\title{
Splenectomy for ABO-Incompatible Kidney Transplantation and Very Late-Onset Cytomegalovirus Disease
}

Tomoaki Iwai, Junji Uchida, Akihiro Kosoku, Kazuya

Kabei, Shunji Nishide, Toshihide Naganuma, Keiko

Maeda, Yuki Yoshikawa, Norihiko Kumada, Yoshiaki

Takemoto, Tatsuya Nakatani

\begin{tabular}{|c|l|}
\hline Citation & Urologia Internationalis, 104(7-8); 651-656 \\
\hline Issue Date & $2020-08$ \\
\hline Published & $2020-05-19$ \\
\hline Type & Journal Article \\
\hline Textversion & Author \\
\hline Rights & $\begin{array}{l}\text { This is the peer-reviewed but unedited manuscript version of the following } \\
\text { article: Urologia Internationalis, } 104(7-8) ; 651-656 . \text { The final, published version } \\
\text { is available at https://doi.org/10.1159/000504862. }\end{array}$ \\
\hline DOI & $10.1159 / 000504862$ \\
\hline
\end{tabular}

Self-Archiving by Author(s)

Placed on: Osaka City University Repository

IWAI, T., et al. (2020). Splenectomy for ABO-Incompatible Kidney Transplantation and Very Late-Onset Cytomegalovirus Disease. Urologia Internationalis. 104, 651-656. 


\section{Case report \\ Splenectomy for ABO-incompatible kidney transplantation and very late-onset cytomegalovirus disease}

Tomoaki Iwai ${ }^{1}$, Junji Uchida ${ }^{1}$, Akihiro Kosoku ${ }^{1}$, Kazuya Kabei ${ }^{1}$, Shunji Nishide ${ }^{1}$, Toshihide Naganuma ${ }^{1}$, Keiko Maeda ${ }^{2}$, Yuki Yoshikawa ${ }^{3}$, Norihiko Kumada ${ }^{4}$, Yoshiaki Takemoto ${ }^{1}$, Tatsuya Nakatani ${ }^{1}$

${ }^{1}$ Department of Urology, Osaka City University Graduate School of Medicine, Osaka, Japan

${ }^{2}$ Department of Nursing, Osaka City University Hospital, Osaka, Japan

${ }^{3}$ Department of Nursing, Shitennoji University, Habikino, Japan

${ }^{4}$ Department of Urology, Suita Municipal Hospital, Suita, Japan

Short Title: Splenectomy and cytomegalovirus disease

*Corresponding Author

Junji Uchida, MD, PhD

Department of Urology

Osaka City University Graduate School of Medicine

1-4-3, Asahi-machi, Abeno-ku, Osaka, 545-8585, Japan

Tel: $+81-6-6645-3857$

Fax: $+81-6-6647-4426$

E-mail: m9492120@msic.med.osaka-cu.ac.jp

Keywords: kidney transplantation, $\mathrm{ABO}$-incompatible, splenectomy, very late-onset, cytomegalovirus 


\begin{abstract}
Introduction

Splenectomy had been previously performed in ABO-incompatible kidney transplantation to reduce the B cell pool. However, studies have shown that patients undergoing splenectomy may have a lifelong susceptibility to infection and mortality. Splenectomy may affect the incidence of cytomegalovirus (CMV) disease even at a very late stage after transplantation in ABO-incompatible recipients.
\end{abstract}

Patients and Methods

Seven patients received their graft from an $\mathrm{ABO}$-incompatible living donor at our institution and underwent splenectomy for $B$ cell reduction. Among them, 3 recipients experienced very late-onset CMV disease approximately 10 years after their transplant and were enrolled in this study.

Results

Very late-onset CMV disease occurred at 9 years and 9 months, 15 years, and 13 years and 5 months after transplantation, respectively. Two recipients suffered from CMV retinitis, while one experienced colitis. The age of the patients at onset of CMV disease was 69 years, 42 years, and 71 years, respectively.

Conclusion

This may be the first report on very late-onset CMV disease after splenectomy in ABO-incompatible kidney transplantation. We should be aware that these recipients can experience very late-onset CMV disease even approximately 10 years after their transplant. 


\section{Introduction}

In Japan, ABO-incompatible kidney transplantation has become a popular alternative to deceased kidney transplantation due to the shortage of deceased donors [1,2]. Splenectomy had been performed to reduce the $\mathrm{B}$ cell pool in $\mathrm{ABO}$-incompatible kidney transplant recipients but is now being replaced by the administration of rituximab, an anti-CD20 antibody [3-5]. During the early era of ABO-incompatible kidney transplantation in Japan, splenectomy had been performed in ABOincompatible kidney transplant recipients because Japanese insurance had not covered administration of rituximab for kidney transplantation. As a result, nowadays, there is a certain number of long-term graft survived recipients without spleen.

A previous study had demonstrated the benefits of splenectomy not only for graft survival but also for patient survival in kidney transplant recipients [6]. However, splenectomy has been reported to be associated with a lifelong increased susceptibility to infection and mortality [7]. Although the risk of infection after splenectomy varies with age, reason for surgery, and length of time after surgery, the extent and magnitude of this risk in these patients have not been well defined. In fact, the relationship between splenectomy and infectious disease in ABO-incompatible kidney transplant recipients undergoing immunosuppressive therapy remains unclear.

Cytomegalovirus (CMV) is the most dangerous viral pathogen after kidney transplants, and CMV disease usually occurs during the first 3 months of transplantation [8]. Reports have been made on very late-onset CMV disease that developed appropriately 10 years after transplantation [9]. Splenectomy may therefore affect the incidence of CMV disease at a very late stage in $\mathrm{ABO}$ incompatible kidney transplant recipients. In this study, we summarized our results on very lateonset CMV disease in recipients who had undergone splenectomy as desensitization for ABOincompatible kidney transplantation at our institution.

\section{Materials and Methods}

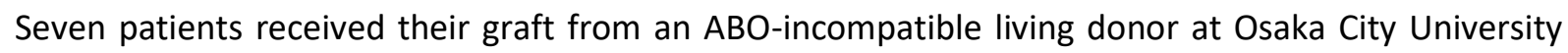
Hospital and underwent splenectomy as B cell reduction between January 1999 and May 2006. Among them, 3 recipients experienced very late-onset CMV disease approximately 10 years after their transplant and were enrolled in this study. All 3 patients were followed until March 2019. The age at transplant was 59, 28, and 57 years, respectively. The patients had initial anti-A/B antibody titers ranging from 1:8 to 1:256. The patient data are given in Table 1.

Our study was approved by the Ethics Committee of Osaka City University Graduate School of Medicine (No. 3957). Opt-out consent was obtained instead of written informed consent. We provided patients with information explaining the proposed research plan (the purpose, required individual data, and duration of research) by means of an information website of our hospital and 
gave them the opportunity of opt-out. All the procedures were in accordance with the Helsinki Declaration of 2000. 


\section{Immunosuppressive protocol}

To remove the anti-A/B antibodies, the patients underwent 4-6 sessions of double-filtration plasmapheresis and plasma exchange prior to kidney transplantation until the anti-A/B titers were less than 1:16. Splenectomy was performed at the time of kidney transplantation. The protocol for ABO-incompatible kidney transplantation with splenectomy is shown in Figure 1[4]. The pretransplant immunosuppressive protocol included B-lymphocyte suppression with 1 week of an antimetabolite. For postoperative immunosuppression, the same regimen as $\mathrm{ABO}$-compatible cases was followed, in which calcineurin inhibitors were initiated 3 days before transplantation combined with two doses of basiliximab. Calcineurin inhibitors (tacrolimus or cyclosporin) were administered at a dose level that resulted in a blood trough level of $13-18 \mathrm{ng} / \mathrm{mL}$ (tacrolimus) or $250-300 \mathrm{mg} / \mathrm{dL}$ (cyclosporin). Basiliximab or anti-lymphocyte globulin was administered for induction immunosuppressive therapy. Maintenance immunosuppression included a calcineurin inhibitor (target trough level of tacrolimus or cyclosporin; $3-5 \mathrm{ng} / \mathrm{mL}$ or $50-100 \mathrm{mg} / \mathrm{dL}$, respectively), mycophenolate mofetil, and methylprednisolone.

For treatment of acute cellular rejection episodes, methylprednisolone was administered at a dose of $500 \mathrm{mg} /$ day for 3 days alone or in combination with deoxyspergualin $(5 \mathrm{mg} / \mathrm{kg} /$ day: $5-7$ days). When resistance to these drugs was confirmed, OKT-3 was administered. Acute cellular rejection and antibody-mediated rejection were histologically diagnosed by light microscopy of graft biopsy specimens according to the Banff criteria.

\section{Diagnosis of CMV infection}

CMV infection was routinely diagnosed and monitored using the C7HRP antigenemia assay once a month until 1 year after kidney transplantation. Recipients were diagnosed with CMV infection in the presence of 5 or more infected cells $/ 5 \times 10^{4}$ leukocytes. CMV disease was diagnosed in patients with at least one of the following: $\mathrm{CMV}$ infection and one or more of the following: fever $\left(>38.0^{\circ} \mathrm{C}\right)$, newonset malaise, leukopenia, atypical lymphocytosis, thrombocytopenia, elevation of hepatic transaminases, or localized CMV infection in a biopsy. These definitions of CMV infection and disease are consistent with the American Society of Transplantation guidelines [10].

\section{Results}

\section{Baseline characteristics}

All 3 patients had received a graft from their spouses. Two patients underwent biopsy-proven acute cellular rejection within 1 year of transplantation. All patients were CMV-seropositive before transplantation. They all had experienced CMV infection as CMV viremia and treated with 
administration of ganciclovir within 1 year of transplantation. The patient characteristics are shown in Table 1.

Very late-onset CMV disease

Very late-onset CMV disease occurred at 9 years and 9 months, 15 years, and 13 years and 5 months after transplantation, respectively. Two recipients suffered from CMV retinitis, while one experienced colitis. Patient 1 had nephelopsia, patient 2 suffered from intractable diarrhea and melena, and patient 3 had nephelopsia and reduced visual acuity. The age of the patients at onset of CMV disease was 69 years, 42 years, and 71 years, respectively, and C7HRP levels at onset of CMV disease were 72,2 , and 16 infected cells/50,000 leukocytes, respectively. One was accompanied by pneumocystis pneumonitis due to pneumocystis jirovecii with CMV retinitis, and another had ulcerative colitis with CMV colitis. Two patients received tacrolimus, mycophenolate mofetil, and steroid, and one received cyclosporine, mycophenolate mofetil, and steroid (Table 2).

Treatment of very late-onset CMV disease

All 3 recipients who experienced CMV disease were treated with administration of ganciclovir at a dose of $5 \mathrm{mg} / \mathrm{kg}$ weight. Patient 1 and 2 underwent additional granulocyte and monocyte adsorptive apheresis to reduce pro-inflammatory cytokines reactivating CMV and to remove CMV-permissive cells. All recipients were successfully treated with administration of ganciclovir in combination with granulocyte and monocyte adsorptive apheresis or only administration of gancyclovir. Patient 1 who had pneumocystis pneumonia was treated with additional administration of sulfamethoxazole/trimethoprim. Patient 2 received salazosulfapyridine for treatment of ulcerative colitis (Table 3 ).

\section{Outcome}

One recipient who suffered from CMV colitis was reintroduced to hemodialysis therapy. The other 2 patients did not experience graft loss or death after transplantation and have maintained good graft functions. However, two patients underwent recurrence of CMV disease, and the current serum creatinine level was 1.2 and $1.3 \mathrm{mg} / \mathrm{dL}$, respectively. The observation period after very late-onset CMV disease was 5 years and 1 month, 3 years and 6 months, and 1 year and 4 months, respectively (Table 3).

\section{Discussion/Conclusion}

The present study demonstrated very late-onset CMV disease after splenectomy in ABOincompatible kidney transplantation. Splenectomy had been performed to reduce B cells in recipients 
of ABO-incompatible kidney transplantation. However, approximately 10 years after their transplant, 3 out of our 7 patients who had received splenectomy suffered from very late-onset CMV disease including retinitis and colitis. Although the sample size of this study was small, our results suggested that splenectomy may be involved in the pathogenesis of very late-onset CMV disease after kidney transplantation. Even to this day, splenectomy may be performed for patients who cannot received rituximab due to such as allergy for rituximab in ABO-incompatible kidney transplantation. This may be the first demonstration of very late-onset CMV disease after splenectomy in ABO-incompatible kidney transplantation. We should be aware that very late-onset CMV disease can occur in recipients of $A B O$-incompatible kidney transplantation with splenectomy even approximately 10 years after their transplant.

CMV infection is associated with significantly decreased kidney transplant patient and graft survivals $[8,11]$. The direct effects of CMV may present as a viral syndrome or tissue-invasive disease [12], and the indirect effects may result in opportunistic infection, acute and chronic graft rejection, graft loss, and decreased recipient survival [13]. The risk is highest in the first few months after transplantation, which is increased by treatment of an acute rejection episode [14]. While antiviral prophylaxis for 3 to 6 months after transplantation has effectively prevented early-onset CMV, it has been reported that approximately $18 \%$ to $31 \%$ of kidney transplant recipients develop late-onset CMV infection or disease after prophylaxis cessation. Late-onset $\mathrm{CMV}$ infection at 6 months to 1 year after transplant is most frequently observed in high-risk CMV-seronegative recipients of an organ from a CMVseropositive donor [15]. However, very late-onset CMV infection at more than 3.5 years following transplantation is not significantly associated with traditional risk factors such as donor seropositive/recipient seronegative status, rejection, induction therapy, and mycophenolate mofetil. Moreover, very late-onset CMV disease is not associated with antiviral prophylaxis [16]. The risk factors of very late-onset CMV disease remains unestablished, but immunosuppressive therapy after $\mathrm{ABO}$-incompatible kidney transplantation with splenectomy may be a risk for very late-onset $\mathrm{CMV}$ disease.

During the early era of $\mathrm{ABO}$-incompatible kidney transplantation, the higher trough concentrations of calcineurin inhibitors for this procedure had been applied because of the possible high risk procedure for rejection compared with ABO-compatible kidney transplantation. Although ABO-incompatible kidney transplantation is an immunological high-risk procedure due to anti-A/B antibodies, a previous study showed no significant differences in frequency of CMV infection between $\mathrm{ABO}$ incompatible and $\mathrm{ABO}$-compatible kidney transplant recipients [2]. However, the risk of $\mathrm{CMV}$ infection after $\mathrm{ABO}$-incompatible kidney transplantation was significantly higher than that after $\mathrm{ABO}$ - 
compatible kidney transplantation in patients who received a non-rituximab-based desensitization protocol [17]. In our study, 3 out of 7 patients who had received ABO-incompatible kidney transplantation with splenectomy and without administration of rituximab as a desensitization protocol developed very late-onset $\mathrm{CMV}$ disease. $\mathrm{ABO}$-incompatible kidney transplantation with a non-rituximab-based desensitization protocol may therefore be high risk not only for usual CMV infection but also for very late-onset CMV disease, which has not been previously reported.

It appears that a relatively higher proportion of very late-onset CMV disease is tissue-invasive. Gastrointestinal disease and retinitis are also more commonly observed with very late-onset CMV disease $[18,19]$. Delayed diagnosis of CMV disease is one potential explanation for the more severe manifestations of very late-onset CMV disease. CMV as a direct cause of death may be more commonly observed in very late-onset CMV disease, because of lack of clinical suspicion or delayed diagnosis [16]. A previous report demonstrated that 3 out of 5 cases of very late-onset CMV disease that occurred 3 to 8 years after transplantation died because of lack of early clinical recognition [20]. If signs of very late-onset CMV disease such as fever, diarrhea, or nephelopsia are recognized, elective further examination, such as C7HRP antigenemia assay or biopsy of the target organ, for diagnosis of $\mathrm{CMV}$ infection may be needed for early detection of CMV infection.

The mechanism of CMV reactivation is generally believed to involve local expression of a wide variety of pro-inflammatory cytokines that play a crucial role in recruiting immune cells, including monocytes, to inflammatory organs. Pro-inflammatory cytokines such as interferon $\gamma$ and tumor necrosis factor $\alpha$ promote reactivation of $\mathrm{CMV}$ in monocytes and dendritic cells. Stimulated by the release of these cytokines and chemokines from inflammatory organs, latently-infected circulating monocytes and dendric cells likely migrate to these sites and differentiate into permissive cells, supporting active replication of CMV [21]. Granulocyte and monocyte adsorptive apheresis is a widely used treatment for inflammatory bowel disease in Japan [22], which may reduce pro-inflammatory cytokine production and decrease the number of monocytes positive for CD14, CD16, and DR subsets. Our previous study suggested that a combination of granulocyte and monocyte adsorptive apheresis with ganciclovir can shorten the therapeutic duration of CMV disease in kidney transplant recipients without any adverse effects [23]. Currently, intravenous administration of ganciclovir is the recommended standard treatment for CMV disease following solid organ transplantation. In recipients with no evidence of ganciclovir resistance, intravenous administration of ganciclovir has been used successfully to reduce the viral burden [24]. Granulocyte and monocyte adsorptive apheresis with ganciclovir may be an effective therapy for very late-onset CMV disease. 
There might be several limitations in this study because of the small number of cases and the fact that is a retrospective study. However, there has been no report on very late-onset CMV disease after splenectomy in ABO-incompatible kidney transplantation. This study may be the first demonstration that splenectomy may be involved in the pathogenesis of very late-onset CMV disease after transplantation. To confirm these findings, further prospective cohort trials with a larger number of patients are needed in future.

In conclusion, the present study demonstrated very late-onset CMV disease after splenectomy for $\mathrm{ABO}$-incompatible kidney transplantation. We should be aware that very late-onset $\mathrm{CMV}$ disease can occur in recipients of $\mathrm{ABO}$-incompatible kidney transplantation with splenectomy even approximately 10 years after their transplant. 


\section{Statements}

\section{Acknowledgement}

\section{Statement of Ethics}

This study was approved by the Ethics Committee of Osaka City University Graduate School of Medicine (No. 3957). Opt-out consent was obtained instead of written informed consent. We provided patients with information explaining the proposed research plan (the purpose, required individual data, and duration of research) by means of an information website of our hospital and gave them the opportunity of opt-out. All the procedures were in accordance with the Helsinki Declaration of 2000 and the Declaration of Istanbul 2008.

\section{Disclosure Statement}

The authors have no conflicts of interest to declare.

\section{Funding Sources}

There is no funding source in this study. 


\section{Author Contributions}

We would like to note that among the authors, T. Iwai, J. Uchida, A. Kosoku, and T. Nakatani designed the study, and T. Iwai, J. Uchida, and A. Kosoku wrote the paper. J. Uchida, K. Kabei, S. Nishide, T. Naganuma, K. Maeda, Y. Yoshikawa, K. Norihiko, Y. Takemoto, and N. Kumada participated in the patients' follow-up and collected data.

All authors have made significant contributions to this paper and confirm the data to be beneficial for physicians involved in kidney transplantation. 


\section{References (Numerical)}

1: Takahashi K, Saito K. ABO-incompatible kidney transplantation. Transplant Rev (Orlando). 2013 Jan;27(1):1-8.

2: Kosoku A, Uchida J, Nishide S, Kabei K, Shimada H, Iwai T, et al. ABO-incompatible kidney transplantation as a renal replacement therapy-A single low-volume center experience in Japan. PLoS One. 2018 Dec 31;13(12):e0208638.

3: Uchida J, Machida Y, Iwai T, Kuwabara N, Iguchi T, Naganuma T, et al. Clinical outcome of ABOincompatible living unrelated donor kidney transplantation. Urol Int. 2011;86(3):307-14.

4: Uchida J, Kuwabara N, Machida Y, Iwai T, Naganuma T, Kumada N, et al. Excellent outcomes of ABO-incompatible kidney transplantation: a single-center experience. Transplant Proc. 2012 Jan;44(1):204-9.

5: Uchida J, Kosoku A, Kabei K, Nishide S, Shimada H, Iwai T, et al. Clinical Outcomes of ABOIncompatible Kidney Transplantation in Patients with End-Stage Kidney Disease due to Diabetes Nephropathy. Urol Int. 2019;102(3):341-347.

6: Takahashi K, Saito K, Takahara S, Okuyama A, Tanabe K, Toma H, et al. Excellent long-term outcome of ABO-incompatible living donor kidney transplantation in Japan. Am J Transplant. 2004;4(7):1089-96.

7: Kyaw MH, Holmes EM, Toolis F, Wayne B, Chalmers J, Jones IG, et al. Evaluation of severe infection and survival after splenectomy. Am J Med. 2006 Mar;119(3):276.e1-7.

8: Razonable RR, Humar A. AST Infectious Diseases Community of Practice. Cytomegalovirus in solid organ transplantation. Am J Transplant. 2013 Mar;13 Suppl 4:93-106.

9: Burgan H, Gosteli G, Giovannini M, Lienhard R, Clerc O. Very-late-onset cytomegalovirus disease: a case-report and review of the literature. BMC Res Notes. 2017 Jun 13;10(1):210.

10: American Society of Transplantation (AST). Cytomegalovirus guidelines for the prevention and management of infectious complications of solid organ transplantation. Am J Transplant. 2004;4 (Suppl. 10):51-8.

11: Browne BJ, Young JA, Dunn TB, Matas AJ. The impact of cytomegalovirus infection $\geq 1$ year after primary renal transplantation. Clin Transplant. 2010 Jul-Aug;24(4):572-7.

12: Rubin RH. The pathogenesis and clinical management of cytomegalovirus infection in the organ transplant recipient: the end of the 'silo hypothesis'. Curr Opin Infect Dis. 2007 Aug;20(4):399-407. 
13: Jorgenson MR, Descourouez JL, Cardinale B, Lyu B, Astor BC, Garg N, et al. Risk of opportunistic infection in kidney transplant recipients with cytomegalovirus infection and associated outcomes. Transpl Infect Dis. 2019 Jun;21(3):e13080.

14: Fishman JA, Emery V, Freeman R, Pascual M, Rostaing L, Schlitt HJ, et al. Cytomegalovirus in transplantation - challenging the status quo. Clin Transplant. 2007 Mar-Apr;21(2):149-58.

15: Jamal AJ, Husain S, Li Y, Famure O, Kim SJ. Risk factors for late-onset cytomegalovirus infection or disease in kidney transplant recipients. Transplantation. 2014 Mar 15;97(5):569-75.

16: Razonable RR, Blumberg EA. It's not too late: a proposal to standardize the terminology of "lateonset" cytomegalovirus infection and disease in solid organ transplant recipients. Transpl Infect Dis. 2015 Dec;17(6):779-84.

17: Scurt FG, Ewert L, Mertens PR, Haller H, Schmidt BMW, Chatzikyrkou C. Clinical outcomes after ABO-incompatible renal transplantation: a systematic review and meta-analysis. Lancet. 2019 May 18;393(10185):2059-2072.

18: Shibolet O, Ilan Y, Kalish Y, Safadi R, Ashur Y, Eid A, et al. Late cytomegalovirus disease following liver transplantation. Transpl Int. 2003 Dec;16(12):861-5.

19: Blyth D, Lee I, Sims KD, Gasink LB, Barton TD, Van Deerlin VM, et al. Risk factors and clinical outcomes of cytomegalovirus disease occurring more than one year post solid organ transplantation. Transpl Infect Dis. 2012 Apr;14(2):149-55.

20: Boehler A, Schaffner A, Salomon F, Keusch G. Cytomegalovirus disease of late onset following renal transplantation: a potentially fatal entity. Scand J Infect Dis. 1994;26(4):369-73.

21: Gandhi MK, Khanna R. Human cytomegalovirus: clinical aspects, immune regulation, and emerging treatments. Lancet Infect Dis. 2004 Dec;4(12):725-38.

22: Sawada K, Muto T, Shimoyama T, Satomi M, Sawada T, Nagawa H, et al. Multicenter randomized controlled trial for the treatment of ulcerative colitis with a leukocytapheresis column. Curr Pharm Des. 2003;9(4):307-21.

23: Naganuma T, Takemoto Y, Iwai T, Kuwabara N, Uchida J, Nakatani T, et al. Effects of Granulocyte and Monocyte Adsorptive Apheresis in Renal Transplantation Recipients With Concomitant Cytomegalovirus Infection. Transplant Proc. 2016 Apr;48(3):929-32.

24: Preiksaitis JK, Brennan DC, Fishman J, Allen U. Canadian society of transplantation consensus workshop on cytomegalovirus management in solid organ transplantation final report. Am J Transplant. 2005 Feb;5(2):218-27. 


\section{Figure Legends}

Fig. 1. Immunosuppressive protocol for ABO-incompatible kidney transplantation with splenectomy.

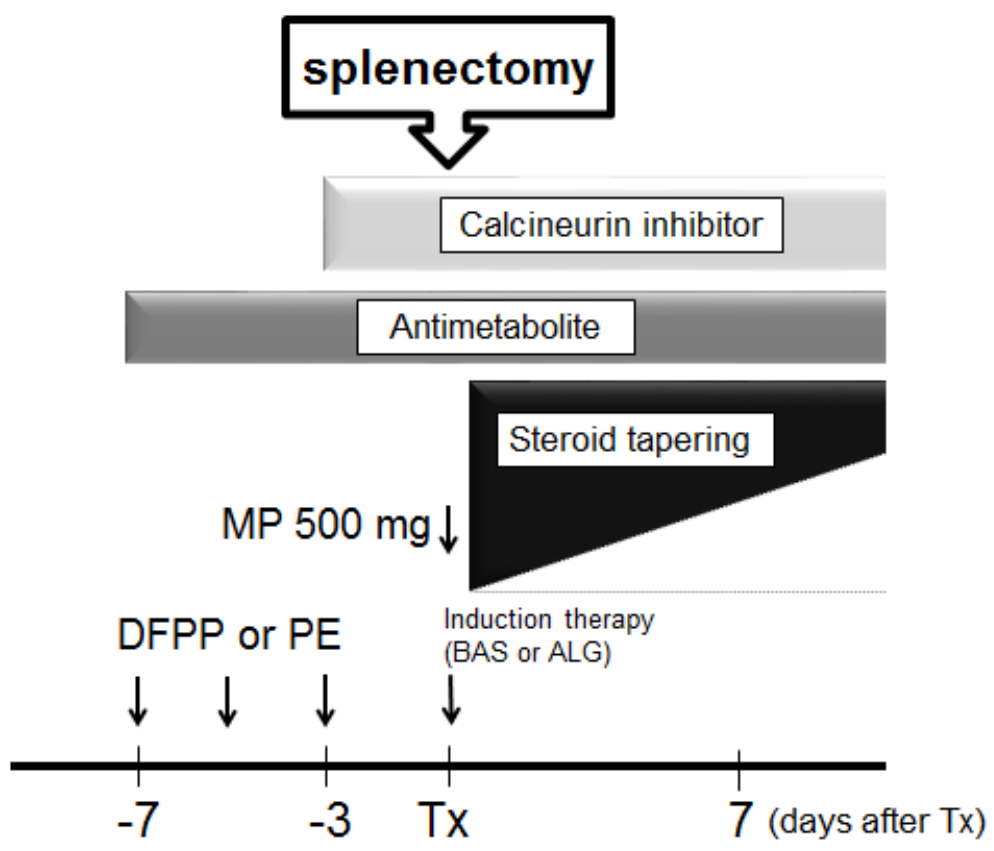


Table 1. Patient characteristics at transplantation.

\begin{tabular}{|c|c|c|c|}
\hline Case & 1 & 2 & 3 \\
\hline Age (year) & 59 & 28 & 57 \\
\hline Sex & male & female & male \\
\hline Cause of kidney disease & $\begin{array}{l}\text { chronic } \\
\text { glomerulonephritis }\end{array}$ & IgA nephropathy & $\begin{array}{l}\text { chronic } \\
\text { glomerulonephritis }\end{array}$ \\
\hline Hemodialysis duration (months) & 32 & 24 & 53 \\
\hline Donor relation & wife & father & wife \\
\hline ABO blood type (donor / recipient) & $\mathrm{A} / \mathrm{O}$ & $A B / A$ & $A B / A$ \\
\hline HLA mismatches (-A, -B, -DR) & $5 / 6$ & $3 / 6$ & $5 / 6$ \\
\hline $\begin{array}{l}\text { Initial anti-A/B antibody titers } \\
\text { (IgG / IgM) }\end{array}$ & $1: 256 / 1: 16$ & $1: 16 / 1: 16$ & $1: 8 / 1: 8$ \\
\hline $\begin{array}{l}\text { Initial flow cytometry crossmatch with } \\
\text { ( } \mathrm{T} / \mathrm{B} \text { cells) }\end{array}$ & $-1-$ & $-/-$ & $-1-$ \\
\hline Desensitization & $\begin{array}{c}\text { DFPP } x 5 \\
\text { PE x1 } \\
\text { splenectomy }\end{array}$ & $\begin{array}{c}\text { DFPP x3 } \\
\text { PE x1 } \\
\text { splenectomy }\end{array}$ & $\begin{array}{c}\text { DFPP x3 } \\
\text { PE x1 } \\
\text { splenectomy }\end{array}$ \\
\hline Induction immunosuppressive therapy & BAS, MP, MMF, Tac & ALG, $\mathrm{Pr}, \mathrm{AZ}, \mathrm{Tac}$ & BAS, MP, MMF, CsA \\
\hline $\begin{array}{l}\text { Acute rejection within } 1 \text { year after Tx } \\
\text { (treatment) }\end{array}$ & $\begin{array}{c}+ \\
\text { (steroid pulse) }\end{array}$ & $\begin{array}{c}+ \\
\text { (steroid pulse) }\end{array}$ & - \\
\hline CMV serostatus before $\mathrm{Tx}$ & + & + & + \\
\hline CMV viremia within 1 year after Tx & + & + & + \\
\hline
\end{tabular}

HLA, human leukocyte antigen; DFPP, double filtration plasmapheresis; PE, plasma exchange; BAS, basiliximab; MP, methylprednisolone; MMF, mycophenolate mofetil; Pr, prednisolone; Tac, 
tacrolimus; CsA, cyclosporine A; ALG, antilymphocyte globulin; AZ, azathioprine; Tx, transplantation; CMV, cytomegalovirus. 
Table 2. Characteristics at very late-onset CMV disease.

\begin{tabular}{|c|c|c|c|}
\hline Case & 1 & 2 & 3 \\
\hline Age at onset (years) & 69 & 42 & 71 \\
\hline Symptoms & nephelopsia & diarrhea, melena & $\begin{array}{l}\text { nephelopsia, } \\
\text { reduced visual } \\
\text { acuity }\end{array}$ \\
\hline CMV disease & $\mathrm{CMV}$ retinitis & CMV colitis & $\mathrm{CMV}$ retinitis \\
\hline $\begin{array}{c}\text { Period from transplantation to } \\
\text { onset }\end{array}$ & $\begin{array}{l}9 \text { years and } 9 \\
\text { months }\end{array}$ & 15 years & $\begin{array}{l}13 \text { years and } 5 \\
\text { months }\end{array}$ \\
\hline $\begin{array}{c}\text { C7HRP (infected cells } / 5 \times 10^{4} \\
\text { leukocytes) }\end{array}$ & 72 & 2 & 16 \\
\hline $\begin{array}{c}\text { Immunosuppressive therapy at } \\
\text { onset }\end{array}$ & MP, MMF, Tac & Pr, MMF, Tac & MP, MMF, CsA \\
\hline Comorbidity & PCP & $\begin{array}{l}\text { IgA nephropathy } \\
\text { recurrence } \\
\text { ulcerative colitis }\end{array}$ & diabetes mellitus \\
\hline
\end{tabular}

CMV, cytomegalovirus; MP, methylprednisolone; MMF, mycophenolate mofetil; Pr, prednisolone;

Tac, tacrolimus; CsA, cyclosporine A; PCP, pneumocystis pneumonia. 
Table 3. Treatments of very late-onset CMV disease and outcomes.

\begin{tabular}{|c|c|c|c|}
\hline Case & 1 & 2 & 3 \\
\hline Treatment $\mathrm{CMV}$ disease & $\begin{array}{l}\text { GCV } \\
\text { GCAP }\end{array}$ & $\begin{array}{l}\text { GCV } \\
\text { GCAP }\end{array}$ & GCV \\
\hline Recurrence of CMV disease & $\begin{array}{c}\text { once } \\
\text { (after half month) }\end{array}$ & $\begin{array}{c}\text { twice } \\
\text { (after } 3 \text { and } 5 \text { months) }\end{array}$ & - \\
\hline Patient survival & + & + & + \\
\hline S-Cr at onset $(\mathrm{mg} / \mathrm{dL})$ & 1.5 & 3.8 & 1.3 \\
\hline $\mathrm{S}-\mathrm{Cr}$ at remission $(\mathrm{mg} / \mathrm{dL})$ & 1.2 & 10.3 (graft loss) & 1.3 \\
\hline $\begin{array}{c}\text { Observation period after } \mathrm{CMV} \\
\text { disease }\end{array}$ & 5 years and 1 month & 3 years and 6 months & 1 year and 4 months \\
\hline
\end{tabular}

CMV, cytomegalovirus; GCV, ganciclovir; GCAP, granulocytapheresis; S-Cr, serum creatinine levels. 